\title{
Segmentation Framework on Digital Microscope Images for Acute Lymphoblastic Leukemia Diagnosis based on HSV Color Space
}

\author{
Kamal A. \\ EIDahshan \\ Professor of Computer \\ Science, Dept. of \\ Mathematics, Faculty \\ of Science, \\ AL-AZHAR University, \\ Cairo, Egypt
}

\author{
Mohammed I. \\ Youssef \\ Dept. of Electronic \\ Engineering, Faculty of \\ Engineering, \\ AL-AZHAR University, \\ Cairo, Egypt
}

\author{
Emad $\mathrm{H}$. \\ Masameer, Ph.D \\ Assistant Professor of \\ Computer Science, \\ Dept. of Mathematics, \\ Faculty of Science, \\ AL-AZHAR University, \\ Cairo, Egypt
}

\author{
Mohammed A. \\ Mustafa \\ Lecturer assistant, Dept. \\ of Management \\ Information Systems, \\ Modern Academy for \\ Computer Science and \\ Information Technology, \\ Cairo, Egypt
}

\begin{abstract}
Image segmentation is considered the most critical step in image processing and plays a vital role in computer vision especially in the medical field. In this work, the segmentation framework based on the color perception characteristics of eyes for acute lymphoblastic leukemia (ALL) images is proposed to segment each leukemia image into two regions: blasts and background. This work is based on nonlinear transformation of microscope color images from RGB color space to HSV color space. In the HSV color space, hue channel is used as a method in segmentation of WBC from its complicated background. The results show that the proposed segmentation framework can differentiate well between normal bone marrow and ALL and become useful for hematologists in further analysis.
\end{abstract}

\section{General Terms}

Medical Image Processing, Image Segmentation, Color Spaces

\section{Keywords}

Microscope Images, Segmentation, ALL, HSV

\section{INTRODUCTION}

Leukemia is a type of cancer caused by abnormal increase of the white blood cells. Yearly thousands of people die of leukemia throughout the world due to the nature of Leukemia cells that become out of control and they spread independently. Early diagnosis and treatment that are applied to the correct cells are vital.

Leukemia can be classified into acute and chronic. Acute leukemia spreads very rapidly and has to be treated promptly rather than chronic leukemia that does not have to be prompt. Acute leukemia can be either lymphoblastic (ALL) or myelogenous (AML), based on affected cell type. Chronic leukemia can be either lymphoblastic (CLL) or myelogenous (CML) [1]. Acute lymphoblastic leukemia (ALL) is considered the prime focus of this work, which has a higher expectation of survival rate compared to AML.

Segmentation is one of the most demanding tasks in image processing. It is used in Computer Vision to automatically partitioning a digital image into a number of different meaningful regions.
For biomedical imaging applications, image segmentation is a founding step in image analysis as it will directly affect the post-processing. It is a crucial component in diagnosis [2] and treatment [3].

The main goal of acute leukemia blood cell segmentation is to extract component such as blast from its complicated blood cells background. There are many techniques that have been developed for image segmentation such as threshold techniques [4], edge detection [5] and watershed techniques [6]. Due to the complex nature of blood cells and overlapping between these cells, segmenting them remains a challenging task [7]. Many algorithms for segmentation have been developed for grayscale images rather than color images which require more information to be processed [8].

In order to simplify enhancement and segmentation for leukemia color images, transformations of original RGB images to different color spaces such as (HSI, HSL, YUV, XYZ, Lab...etc.) are proposed in many works. This transformation reduces correlation between different color channels compared to RGB color space and enables dealing with these channels separately which reduces the complexity of the enhancement and segmentation problems and saves computation time. Based on HIS color space, enhancement technique [9] and Segmentation [10] for leukemia images is proposed. According to [11], Lab color space is used for segmentation process. This work focuses on HSV color space for segmentation as it provides useful chromatic information of the leukemia images [12].

\section{FRAMEWORK}

\subsection{Images Dataset}

Microscope Images of ALL are taken from ALL-IDB database [13]. The images of the database have been captured with an optical laboratory microscope coupled with a Canon Power Shot G5 camera. All images are in JPG format with 24 bit color depth, resolution $2592 \times 1944$. The images are taken with different magnifications of the microscope ranging from 300 to 500. ALL-IDB2 version of the database is used. Figure 1 shows the sample of ALL images. 

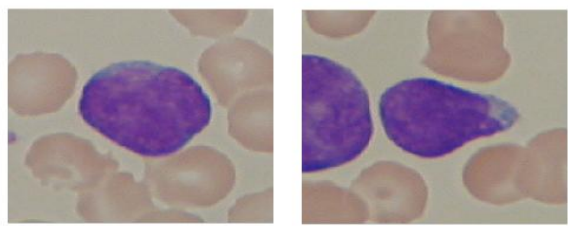

Figure 1: Sample of ALL images

\subsection{Color space conversion}

Due to the inherent interdependence between luminance and chrominance in the RGB color space, a HSV color space is used. HSV provides useful chromatic information of the image. According to [14], HSV color space is familiar to human perception and more suitable for image processing than RGB color space. The representation of HSV color space is shown in figure 2 that shows the cone of HSV model.

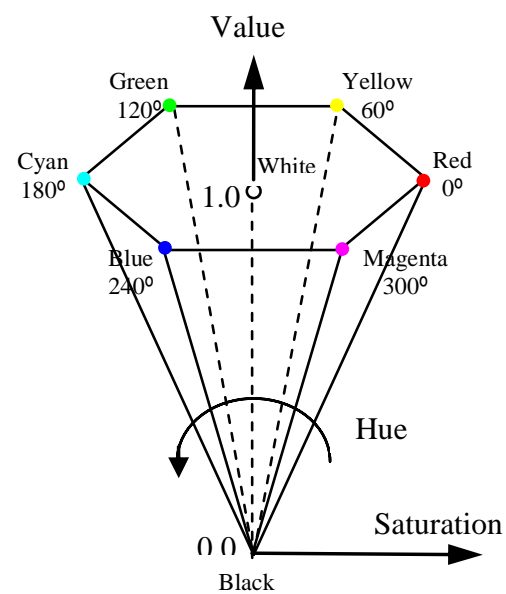

Figure 2: HSV color space

The hue $(\mathrm{H})$ channel refers to the color type such as (Red, Green, Yellow...etc.). The range of hue values changes from $0^{\circ}$ to $360^{\circ}$ passing throw rainbow colors as shown in figure 3 .

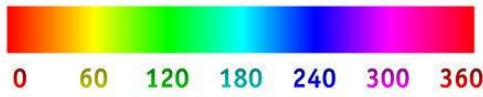

Figure 3: Hue Scale

Table 1 represents the relationship between hue angle and color type. Saturation (S) value affects the purity of the colors while Value (V) means the amount of light in the color. Both $\mathrm{S}$ and $\mathrm{V}$ range from 0 to 1 .

Table 1. Hue angle and color type

\begin{tabular}{|c|c|}
\hline Hue angle & Color type \\
\hline $0^{\circ}$ & Red \\
\hline $60^{\circ}$ & Yellow \\
\hline $120^{\circ}$ & Green \\
\hline $180^{\circ}$ & Cyan \\
\hline $240^{\circ}$ & Blue \\
\hline $300^{\circ}$ & Magenta \\
\hline
\end{tabular}

Transformation the source RGB color space to HSV color space is performed based on the following equations:

$H= \begin{cases}0 & \text { if } M=m \\ \left(60^{\circ} X \frac{g-b}{M-m}+0^{\circ}\right) \bmod 360^{\circ} & \text { if } M=r \\ 60^{\circ} X \frac{b-r}{M-m}+120^{\circ} & \text { if } M=g \\ 60^{\circ} X \frac{r-g}{M-m}+240^{\circ} & \text { if } M=b\end{cases}$

$S=\left\{\begin{array}{l}0 \\ \frac{M-m}{M}=1-\frac{m}{M}\end{array}\right.$

if $M=0$

$V=M$

Where:

$M$ means the maximum values in $\mathrm{R}, \mathrm{G}$, and $\mathrm{B}$ elements. $m$ means the minimum values in $\mathrm{R}, \mathrm{G}$, and $\mathrm{B}$ elements.

\subsection{Proposed approach}

The ultimate goal of ALL segmentation is to extract component such as blast from its complicated blood cells background by using HSV color space.

There are 6 steps involved in applying image segmentation process as shown in figure 4 .

Step 1: transforming the source RGB color space to HSV color space.

Step 2: extracting H channel from HSV color space.

Step 3: Selecting color range of nucleus and cytoplasm by using color histogram of $\mathrm{H}$ channel. Two angle values A1, A2 are obtained from color histogram for segmentation.

Step 4: Implementing the median filter $\mathrm{N} \mathrm{X} \mathrm{N} \mathrm{(N=7)} \mathrm{to} \mathrm{the}$ resulted images.

Step 5: Synthesizing the HSV image.

Step 6: Converting the HSV image to RGB to display. 


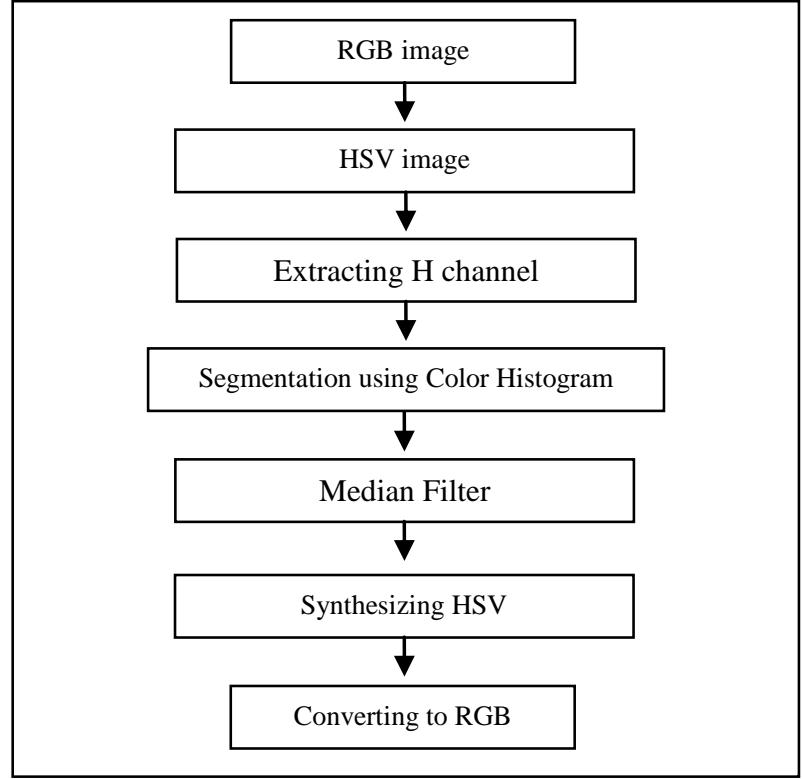

Figure 4: Block diagram of proposed framework

\section{RESULTS AND DISCUSSIONS}

The proposed framework described above is tested on two blood samples of ALL. The results obtained after applying proposed framework are shown as follow:

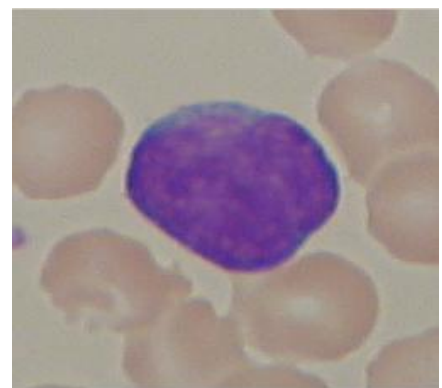

Figure 5: Original RGB image

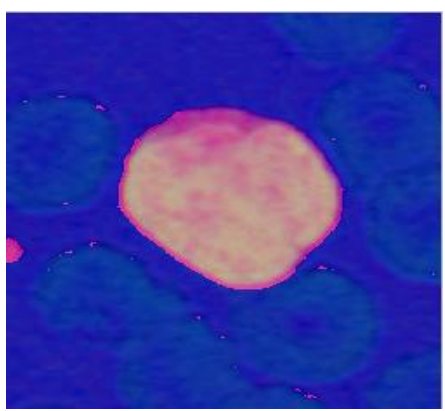

Figure 6: Equivalent HSV image

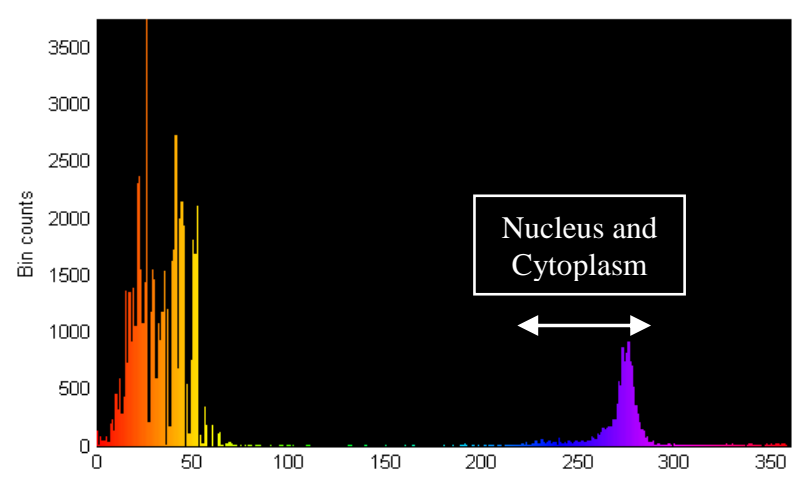

Figure 7: H channel color histogram from resulted image

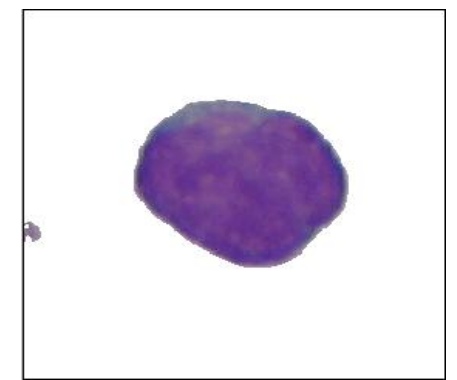

Figure 8: Segmented image

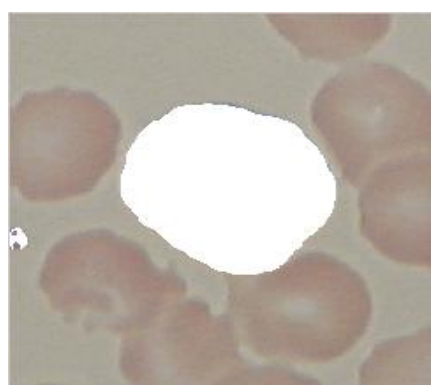

Figure 9: Ghost image for HSV space

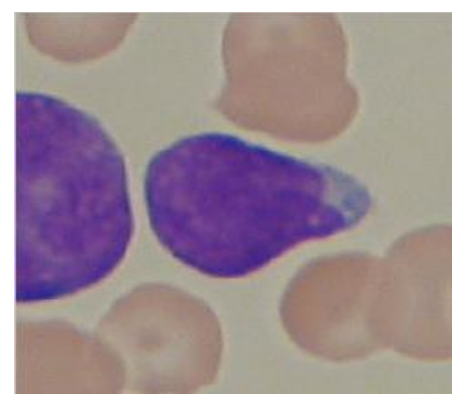

Figure 10: Original RGB image

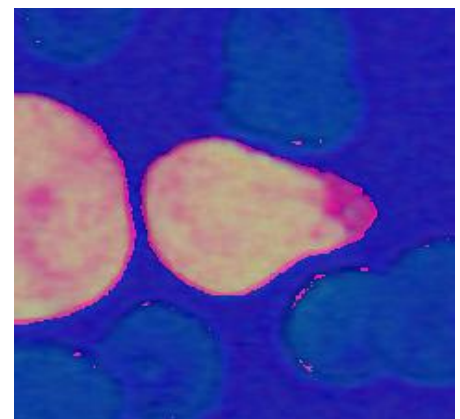

Figure 11: Equivalent HSV image

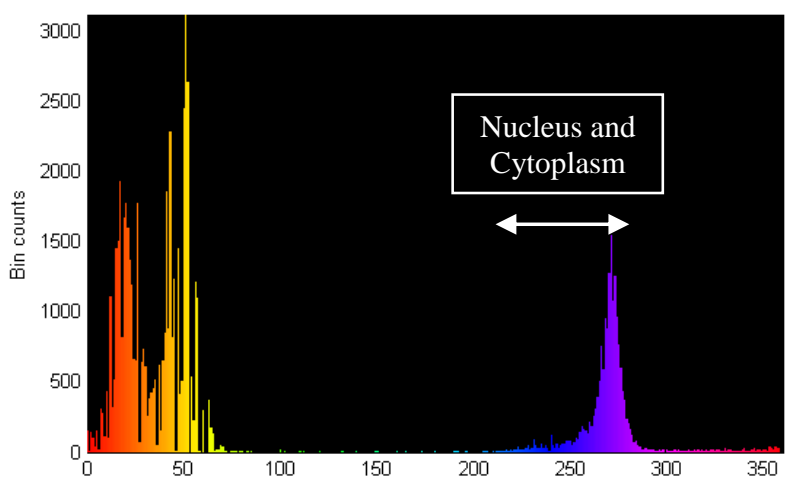

Figure 12: H channel color histogram from resulted image 

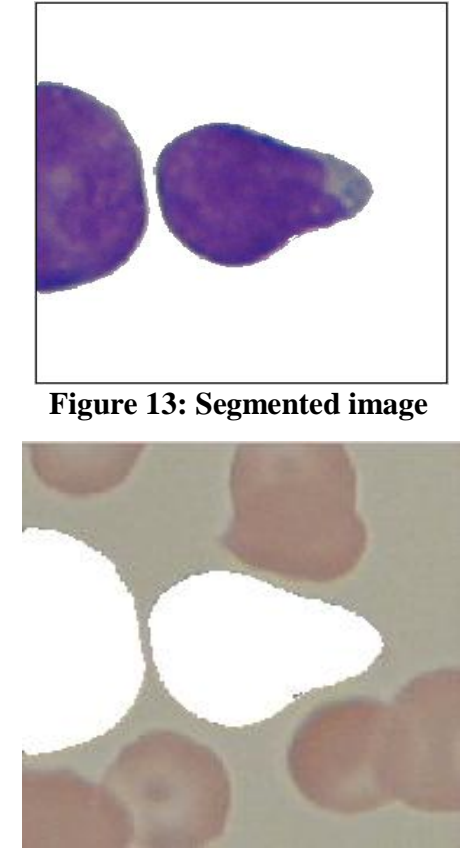

Figure 14: Ghost image for HSV space

Figure 5 and Figure 10 represent the original RGB acute leukemia images. Meanwhile, Figures 6 and Figure 11 show the images after converting to HSV color space. The results obtained in Figure 7 and Figure 12 show that the proposed framework by selecting the range of colors belongs to blast (nucleus and cytoplasm) based on color histogram of $h$ channel.

Figure 8 and Figure 13 represent the output images after segmentation. Also, 7x7 median filter is applied to reduce acquisition noise and staining from images. Finally, Figure 9 and Figure 14 show the ghost image for HSV color space.

\section{CONCLUSION}

By using HSV color space in this work, simplification of the image analysis and processing is obtained. The segmentation framework is based on hue channel of HSV color space. The results obtained from proposed framework yield better accuracy. Also, the blasts of acute lymphoblastic leukemia samples are successfully segmented from its background and unwanted noises. The results also show that the color histogram of $\mathrm{H}$ channel is also useful to select the color range of blasts. In the future, the result of this work can be used as the basis for extracting the other features from the acute lymphoblastic leukemia blood samples.

\section{ACKNOWLEDGMENTS}

The author wishes to thank Prof. Kamal El-Dahshan for his continuous encouragement and support. Prof. Mohammed Ibrahim Youssef for his guidance and valuable suggestions. Dr. Emad Masameer for his help and his valuable advice to complete this paper.

\section{REFERENCES}

[1] G. C. C. Lim. 2002. Overview of Cancer in Malaysia, Japanese Journal of Clinical Oncology, Department of
Radiotherapy and Oncology, Hospital Kuala Lumpur, Kuala Lumpur, Malaysia.

[2] P. Taylor. 1995. Invited review: computer aids for decision-making in diagnostic radiology - a literature review. Brit. J. Radiol.., 68:945-957.

[3] V.S. Khoo, D.P. Dearnaley, D.J. Finnigan, A. Padhani, S.F. Tanner, and M.O. Leach. 1997. Magnetic resonance imaging (MRI): considerations and applications in radiotheraphy treatment planning. Radiother. Oncol., 42:1-15.

[4] S.U. Lee and S.Y. Chung. 1990. A comparative performance study of several global thresholding techniques for segmentation - in Computer Vision, Graphics and Image Processing, no. 52, pp. 171-190.

[5] D. Ziou and S. Tabbone. 1997. Edge detection techniques - An overview- in Technical Report, no. 195, Departement de math et informatique, Universite de Sherbrooke.

[6] L. Vincent and P. Soille. 1991. Watersheds in digital spaces; an efficient algorithm based on immersion simulations - in IEEE Transactions on Pattern Analysis and Machine Intelligence, no. 13, pp. 583-597.

[7] S. Mao-jun, W. Zhao-bin, Z. Hong-juan, and M. Yi-de. 2008. A New Method for Blood Cell Image Segmentation and Counting Based on PCNN and Autowave, in ISCCSP 2008 Malta.

[8] W. Skarbek and A. Koschan. 1994. Colour Image Segmentation, Institute for Technical Informatics, Technical University of Berlin, Berlin.

[9] N. H. A. Halim, M. Y. Mashor, A. S. Abdul Nasir, N. R. Mokhtar, and H. Rosline. 2011. Nucleus segmentation technique for acute leukemia, in Proceedings of the IEEE 7th International Colloquium on Signal Processing and Its Applications (CSPA '11), pp. 192-197, March 2011.

[10] Nor Hazlyna Harun, ,MY Mashor, ,NR.Mokhtar, ,Aimi Salihah, A.N, ,Rosline Hassan, R.A.A. Raof, ,MK Osman.2010. Comparison of Acute Leukemia Image Segmentation using HIS and RGB Color Space, in ISSPA.

[11] Mohapatra S, Patra D. 2010. Automated leukemia detection using hausdorff dimension in blood microscopic images. Proc Int Conf Emerg Trends Robot Commun Technol; 64-8.

[12] Sural, S., G. Qian, and S. Pramanik. 2002. Segmentation and Histogram Generation Using the HSV Color Space for Image Retrieval. In International Conference on Image Processing (ICIP). p. 589-592.

[13] Donida Labati, R., Piuri, V., Scotti, F. 2011. ALL-IDB: the Acute Lymphoblastic Leukemia Image DataBase for image processing.

[14] R. S. Ledley, M. Buas, \& T. J. Colab. 1990. Fundamentals of true-color image processing, Proc. 10th IEEE Conf. on Pattern Recognition, Los Alamos, CA, USA. 